\title{
A Tenerife, Rio de Janeiro, Hawaii quel giorno mancò la comunicazione; è mai successo nei nostri Laboratorie e nelle nostre Sale Operatorie?
}

\section{The communication lacked that day in Tenerife, Rio de Janeiro, Hawaii; has it ever happened in our Laboratories and in our Operating Rooms?}

\author{
Piersandro Sette $\cdot$ Romolo M. Dorizzi \\ Ricevuto: 13 luglio 2013 / Accettato: 19 luglio 2013 / Pubblicato online: 27 agosto 2013 \\ (C) Springer-Verlag Italia 2013
}

Riassunto Il Laboratorio di analisi e la Sala Operatoria sono ambienti complessi più simili tra loro di quanto si possa pensare, perché sono contesti in cui devono essere svolti molti compiti eterogenei e devono interagire molti operatori; le interfacce sono tali da rendere la trasmissione incompleta, inaccurata o ritardata e più operatori concorrono al completamento di un compito, con aumento del numero di interruzioni. Tutte queste caratteristiche sono comuni anche all'ambiente militare, dove un'interruzione o un ritardo possono portare a effetti catastrofici; per minimizzare questo rischio l'intelligence militare ha coniato da molti anni l'acronimo $\mathrm{C}_{4} \mathrm{I}$ (Command, Control, Communication, Computer e Intelligence). In tutti questi contesti gli errori sono inevitabili; ogni strategia tesa alla loro eliminazione o contenimento deve essere implementata.

Uno strumento derivato dall'Aeronautica e da qualche anno utilizzato anche in ambiente sanitario è la checklist di Sala Operatoria. Spesso la cabina di pilotaggio di un aereo, per complessità di lavoro e molteplicità delle figure professionali coinvolte, è paragonata a una Sala Operatoria. Gli incidenti aerei di Tenerife, Rio de Janeiro e l'incidente del sottomarino Greenville alle Hawaii analizzati di seguito hanno in comune proprio la carenza di comunicazione tra gli operatori, carenza che spesso avvertiamo anche nei nostri Laboratori e nelle Sale Operatorie. Oltre al difetto di comunicazione, questi incidenti sono accomunati anche dalla

\section{P. Sette}

UOS Operating Room Management, Ospedale G. Fracastoro, San Bonifacio (VR), Italia

R.M. Dorizzi $(\bowtie)$

UOC Corelab, Laboratorio Unico di AvR, Piazzale della Liberazione 60, 47522, Pievesestina di Cesena (FC), Italia e-mail: rdorizzi@ausl-cesena.emr.it mancanza di leadership, situation awareness, teambuilding e teamworking: le famose non technical skills (NTS) che determinano il successo di un team. Le NTS sono alla base del Crew Resource Management, un insieme di abilità implementato negli anni proprio dai sistemi complessi, prima fra tutti l'aviazione, che, associati alle abilità tecniche, rendono più performanti gli ambienti lavorativi.

Un elemento molto analizzato come causa di errore sia in Sala Operatoria sia in Laboratorio è rappresentato dalle interruzioni. Negli anni recenti si sono prepotentemente imposti nella nostra vita quotidiana gli smartphone, dispositivi che, combinando PDA e telefono cellulare, assicurano un livello di connettività che può diventare universale. A questo riguardo è importante che in Sanità e in Laboratorio si abbiano presenti i "pericoli" degli smartphone, i principali dei quali sono la forte capacità di distrazione del professionista che può indurre errori e il rischio che produca una "dipendenza", la quale può essere dannosa per la salute e in ultima analisi anche per l'organizzazione.

Parole chiave Incidenti aerei - Non-technical skills . Comunicazione - Laboratorio di analisi - Sala Operatoria . Checklist $\cdot$ Smartphone

Summary Laboratory Analysis and Operating Room (OR) are very similar settings, in which operators of different competence carry out different procedures and receive informations from multiple sources. These complex environments can delay information and help frequent interruptions. Surgical checklists modelled after the aeronautical milieu are designed to promote interdisciplinary communication and to provide a framework for the many perioperative steps involved in patient care.

The article shortly analyzes recent aviation and naval disasters (or miracles) (Tenerife, Rio de Janeiro and Hawaii) 
were the lack or the correct use of checklist and non technical skills (NTS) resulted decisive tools in influencing staff communications. Checklists have been used in aviation industry for many years to enhance safety, providing clear support in complex environments. The checklists ensure that all procedures are correctly carried out, eliminating redundant steps and reliance on the inherently fallible human memory. The acronym $\mathrm{C}_{4} \mathrm{I}$, borrowed by military environment, explains how the implementation of the following four skills can limit the risks and errors, also in different fields: Command, Control, Communication, Computer and Intelligence. Also the interruptions can cause mistakes both in Operating rooms and in Laboratory and they are greatly increased by the diffusion of smartphones. It is very important to assure a correct use of these devices and physicians and laboratorians must learn to use them with caution avoiding a smartphone addiction dangerous to the health worker and to the organization.

Keywords Airplane accidents $\cdot$ Non-technical skills . Comunication - Laboratory analysis . Operating room . Checklists $\cdot$ Smartphone

Talvolta siamo tentati di credere che gli eventi altamente improbabili non possano mai accadere, siano impossibili. Tuttavia questi eventi accadono e causano incidenti, spesso tanto più tragici quanto più appaiono improbabili. Gli incidenti gravi avvengono per una combinazione altamente improbabile di eventi cosiddetti impossibili. Vi sono numerosi incidenti che si sono verificati in sistemi complessi come quello dell'Aeronautica e della Navigazione, i quali sono stati analizzati in modo approfondito per comprenderne la dinamica e, soprattutto, individuarne le strategie per evitare che si ripetano e, comunque, limitarne le conseguenze.

I percorsi di certificazione e accreditamento hanno aumentato l'interesse nella ricerca e nella correzione degli errori in un altro sistema complesso e, a volte, complicato come la Medicina, in cui si stanno introducendo metodologie simili a quelle ormai consolidate in Aviazione.

Ma il lavoro non è finito né in Aviazione, né in Medicina.

\section{Miserie e splendori della navigazione aerea e subacquea}

Il 27 marzo 1977, all'aeroporto di Los Rodeos di Tenerife si consumò quello che rimane il più disastroso incidente aereo della storia (583 morti): la collisione in fase di decollo tra due Boeing [1]. L'analisi degli eventi ha dimostrato che l'incidente è risultato da una tragica somma di errori umani, di spazi inadeguati, di situazioni stressanti, di condizioni metereologiche avverse e di comunicazione non adeguata. L'incidente avvenne nel primo pomeriggio di una domenica piovosa e nebbiosa, con visibilità molto ridotta e con una pressione situazionale importante. Infatti, dopo l'esplosione di una bomba rivendicata come attentato terroristico e la minaccia di una seconda esplosione, l' aeroporto internazionale di Las Palmas sull'isola Gran Canaria fu chiuso temporaneamente e tutti i voli internazionali furono deviati sul piccolo aeroporto di Los Rodeos, sulla vicina isola di Tenerife, un aeroporto piccolo, ma con limiti notevoli. Dotato di una sola pista di decollo e una sola via di rullaggio, non era adatto a gestire un numero elevato di voli internazionali e alle manovre degli aerei di linea di grandi dimensioni. Nel piccolo aeroporto erano gestiti brevi voli interni e il personale della torre di controllo conosceva poco l'inglese e comunicava soprattutto in spagnolo.

Gli eventi di quella domenica avevano più che triplicato il traffico aereo e in particolare avevano aumentato i voli internazionali come quello del Boeing 747 Pan American 1736, proveniente dagli Stati Uniti e diretto a Las Palmas, e quello del Boeing 747 KLM 4805, diretto ad Amsterdam, che doveva fare solo uno scalo tecnico. Il pilota del KLM 4805 era il comandante Jacob Velduyzen Van Zenten, con molte migliaia di ore di volo, assegnato ultimamente all'addestramento dei piloti neoassunti al simulatore di volo e a incarichi istituzionali (tanto da essere stato scelto da KLM per una campagna pubblicitaria sulla sicurezza aerea). Van Zenten era atterrato a Tenerife verso le 13 e 10 e aveva fretta di ripartire; stava infatti per scadere il tempo massimo per il limite di servizio e, se superato, l'equipaggio sarebbe stato obbligato a una sosta di riposo. Questa eventualità comportava la necessità di far trascorrere ai passeggeri la notte in albergo a Tenerife a spese della compagnia. Lo scalo tecnico a Los Rodeos non piaceva nemmeno al comandante del Pan American 1736, che aveva chiesto l'autorizzazione di aspettare in volo la riapertura di Las Palmas, ma gli era stata negata e si era visto costretto a uno scalo tecnico nel piccolo aeroporto.

Il volo Pan American 1736 atterrò alle 13 e 45 e parcheggiò a fianco del KLM 4805. Poco dopo, la torre di controllo comunicò la riapertura dell'aeroporto di Las Palmas; Van Zenten decise di prenotare la partenza e di anticipare il rifornimento di carburante a Los Rodeos, manovra che avrebbe ritardato la partenza, ma avrebbe accorciato di molto la successiva sosta a Las Palmas e, soprattutto, avrebbe evitato il fermo di servizio. Le manovre di rifornimento ritardarono il Pan American, che non poteva superare il KLM per la pista troppo stretta. Nel frattempo le condizioni meteorologiche peggioravano; di lì a poco una spessa nebbia avrebbe obbligato a chiudere lo spazio aereo. Quando fu data l'autorizzazione al KLM 4805 di portarsi a fine pista, ruotare di 180 gradi e attendere il via libera per il decollo, la pista non era già più visibile. Il Pan American, che in quel momento era al capo opposto della pista, avrebbe dovuto imboccare un'uscita laterale (taxiway 3 ) per permettere il rullaggio dell' aereo olandese, che la nebbia rendeva difficile da trovare e più difficile ancora da imboccare per un aereo di grandi 
dimensioni come il Boeing 747. Mentre il Pan American ingombrava ancora la pista (anche se invisibile per la nebbia), Van Zenten iniziò le manovre di decollo del suo Boeing 747. Il primo ufficiale, addetto alle comunicazioni radio, informò il capitano della mancata autorizzazione per la rotta; Van Zenten ridusse solamente la potenza dei motori e, appena la torre di controllo comunicò la rotta strumentale, ritenne che l'autorizzazione al decollo fosse implicita e iniziò il decollo non autorizzato. Il primo ufficiale del KLM comunicò di essere al decollo, ma la torre di controllo non capì che stava per decollare e si limitò a ripetere che avrebbe comunicato successivamente l'autorizzazione. Tutta la conversazione fu sentita anche dall'equipaggio del PAM 1736, che stava ancora cercando l'uscita indicatagli e che segnalò la sua presenza sulla pista; la torre di controllo rispose di liberare la pista. L'equipaggio del KLM 4805 era talmente concentrato sulla manovra di decollo che solo il tecnico di volo prestò attenzione alla segnalazione dell'aereo americano in manovra sulla pista e chiese per due volte al comandante se il Pan American avesse liberato la pista. Nessuno ha mai capito perché van Zenten abbia risposto "Yes he did!". Il KLM 4805 continuò il decollo (non visto dalla torre di controllo) e intanto il Pan American aveva appena mancato la taxiway 3 e si trovava di fronte al KLM. Quando il comandante dell'aereo olandese si rese conto dell'impatto imminente cercò di decollare passando sopra al PAM 1736, ma il carrello del KLM scoperchiò la fusoliera del PAM, su cui si sparse una pioggia di scintille che innescò l'esplosione dei serbatoi degli aerei, entrambi a pieno carico.

Il disastro è stato studiato e analizzato con un' accuratezza straordinaria ed è possibile trovare sul web una documentazione eccezionalmente accurata al riguardo [2]. Per sintetizzare le risultanze a cui sono pervenute la autorità competenti internazionali e delle Nazioni maggiormente coinvolte, il disastro ebbe alcune cause principali:

- l'autonomia decisionale di ogni professionista presente nel cockpit, annullata dalla personalità e dal forte carisma del comandante Van Zenten, era diventata la situazione contraria, eteronomia, in cui viene meno quella sorta di intelligenza collettiva che governa i gruppi ben affiatati e il singolo diventa l'esecutore dei desideri di un altro di personalità più forte;

- l'utilizzo di un linguaggio tecnico non standardizzato e la conoscenza imperfetta della lingua inglese da parte della torre di controllo concorsero a determinare l'errore umano;

- le avverse condizioni meteorologiche sulla pista (per altro tipiche a Los Rodeos);

- la concentrazione eccezionale del traffico aereo su un aeroporto non idoneo;

- le inappropriate indicazioni date dalla torre di controllo al PAM 1736 di imboccare un'uscita eseguendo una manovra pressoché impossibile per Boeing 747.

\section{La nascita delle checklist}

Un altro disastro che ha segnato profondamente la storia della Boeing si è verificato quarant'anni prima, il 30 ottobre 1935, quando il Model 299, un innovativo quadrimotore con una capacità di carico e autonomia doppie rispetto agli altri velivoli, partecipò alla selezione del nuovo cacciabombardiere per l'aviazione americana che si svolse sul campo militare di Dayton. La Boeing concorreva con la sua "Fortezza volante" ed era favoritissima, ma un incidente costò la vita a due dei cinque membri dell'equipaggio, tra cui il leggendario comandante Ployer Peter Hill, e portò l'azienda sull'orlo del fallimento [3]. Sul nuovo prototipo il pilota si doveva occupare di molti strumenti manuali e meccanici e si era semplicemente dimenticato di sbloccare il fermo di rotta. Nonostante le comprensibili diffidenze nei confronti del prototipo, la commissione giudicatrice era fortemente convinta che il Model 299 fosse un ottimo aereo, sicuro e pilotabile, e che si meritasse una prova d'appello. Bisognava trovare un modo per descrivere le diverse procedure nello stesso ordine in cui le operazioni dovevano essere eseguite: nasceva la prima checklist in Aeronautica [4].

Il Model 299 (con la sigla B-17) è stato il primo aereo prodotto in grandi numeri (oltre 12.000 esemplari) e diventò una delle armi più importanti del secondo conflitto mondiale. Le checklist si sono estese a molti altri ambiti e nel 2009 anche l'Organizzazione Mondiale della Sanità (OMS) ha proposto la sua prima checklist per la Sala Operatoria, ambiente molto spesso paragonato a quello aeronautico $[5,6]$.

Neppure una checklist, tuttavia, sarebbe riuscita a scongiurare il disastro del sommergibile atomico USS Greenville, che nel 2001 speronò e affondò il peschereccio giapponese Ehine Maru causando 9 morti durante una manovra di emersione dimostrativa [7]. Il programma della giornata per l'addestramento dell'equipaggio del sommergibile e per un gruppo di ospiti illustri della cerimonia prevedeva un'immersione a grande profondità, una serie di manovre per dimostrarne la grande manovrabilità e una simulazione di emersione in situazione di emergenza. La giornata era incominciata male: la foschia nella zona delle manovre era particolarmente fitta e bianca, il programma della visita aveva accumulato un ritardo di quasi un'ora e il monitor del sonar era guasto. Il comandante decise, comunque, di assumere personalmente il timone nella fase di emersione rapida e, dato il ritardo accumulato, volle ridurre i tempi di verifica da parte dell'operatore sonar sulla presenza di eventuali rischi di collisione durante la manovra: gli 8 minuti dedicati alla procedura standard furono quasi dimezzati. L'operatore sonar identificò un rischio di ostacolo a una distanza stimata di circa 8000 metri, ma non proseguì con le operazioni di verifica; non comunicò neppure al comandante che a una nuova rilevazione effettuata qualche minuto dopo la distanza 
dall'ostacolo era scesa a 4000 metri e non si pose il problema dell'attendibilità delle due rilevazioni così diverse. La variazione della posizione del sommergibile rispetto ai riferimenti circostanti non fu aggiornata e questo impedì ogni ulteriore controllo e soprattutto non permise agli ufficiali di accorgersi della presenza di un'altra imbarcazione di superficie. La manovra di emersione rapida fu ritenuta così sicura che venne annullato il briefing di emersione, una procedura standardizzata in cui il comandante, l'operatore sonar e l'ufficiale di rotta esaminano i dati e valutano la sicurezza della manovra. A quel punto il comandante ordinò l'emersione, limitandosi a un rapido sguardo sulla linea di superficie attraverso il periscopio: la turbolenza della manovra e il colore bianco dell'imbarcazione sovrastante impedirono di vedere il peschereccio Ehime Maru, rendendo inevitabile l'impatto [8].

Anche in questo caso, una sequenza di piccoli errori concatenati ha portato al disastro, nonostante fossero disponibili la migliore tecnologia e il personale meglio addestrato. Se il comune denominatore alla base degli errori considerati è la carenza o l'assenza di comunicazione, il caso dell'incidente del volo Air France AF447, precipitato al largo dell'Atlantico provocando 228 vittime il primo giugno 2009 è addirittura paradigmatico [9]. L'inchiesta, durata 2 anni e basata sulle "due scatole nere" in cui sono incise le voci dei piloti nei drammatici ultimi 15 minuti della loro vita, ha dimostrato che si tratta di un incidente evitabile. La sera del 31 maggio 2009 il volo AF447, un Airbus 330, decollava da Rio de Janeiro diretto a Parigi. L'equipaggio, composto dal comandante, capitano Marc Dubois, con più di 10.000 ore di volo, il giovane primo copilota Pierre-Cédric Bonin, con poca esperienza, e il secondo copilota, David Robert, giovane ma più esperto, sapeva dai bollettini meteo di andare incontro a un'ampia perturbazione. La temperatura si abbassò rapidamente e i sensori esterni si ghiacciarono, disattivando il pilota automatico. Dopo qualche ora di volo, l'AF447 manteneva ancora la rotta iniziale, a differenza di tutti gli altri aerei che avevano chiesto e ottenuto di cambiare rotta. La temperatura esterna s'innalzò improvvisamente, rendendo più rarefatta l'aria e impedendo all'aereo, ancora troppo pesante a causa del pieno di carburante, di sollevarsi al di sopra delle nubi. Poco dopo le 2 del mattino, il comandante prese una pausa e delegò al comando dell'aereo Bonin, il copilota più giovane e meno esperto, che, nonostante il maltempo aumentasse, non studiò la situazione meteo e non chiese la possibilità di spostarsi da quella zona. Nel frattempo il secondo copilota si era accorto che il radar non era stato impostato correttamente e che l'aereo si stava infilando in una zona con perturbazioni ancora più violente. A questo punto si verificava l'errore umano, che da solo innescò tutta la catena di avvenimenti che condanneranno l'aereo: Bonin, probabilmente sotto pressione dalla responsabilità, dalle turbolenze, dal fatto di dover pilotare manualmente, tirò verso di sé la cloche per cercare di prendere quota, ma questo non avvenne per via del peso del carburante e della temperatura relativamente calda. L'aereo rispose al contrario di quanto il copilota si aspettasse, perse potenza e, invece di salire, cominciò a entrare in stallo (che è segnalato da un allarme sonoro e luminoso, non disattivabile, ripetitivo e irritante che non è possibile ignorare). Anche se tutti i piloti sono addestrati a superare lo stallo abbassando la cloche in modo che l'aereo scenda di quota e riacquisti velocità, i piloti dell' Airbus 330 non solo non avvertirono l'allarme (che si attiverà per 75 volte in quei fatali 15 minuti!) ma si comportarono esattamente al contrario di quanto previsto dalle procedure di volo: la cloche venne costantemente tenuta indietro! Dopo qualche minuto subentrò il comandante, che non prese il posto del copilota ma rimase seduto alle sue spalle e non si accorse della posizione della cloche e l'aereo precipitò nell'Oceano qualche minuto dopo che Dubois prese fisicamente il comando. Quello che è apparso immediatamente chiaro alla commissione del Bureau d'Enquêtes et d'Analyses (BEA) è che fin dall'inizio delle difficoltà è venuto meno o è stato dimenticato il Crew Resource Management o CRM. Come nel caso di Tenerife, un forte stress tende a bloccare la parte del cervello deputato all'innovazione e alla creatività e s'instaurano invece meccanismi conosciuti e stereotipati. La situation awareness è andata completamente perduta, non era chiaro chi comandasse l'aereo e che cosa si dovesse fare in assenza del comandante (leadership). Le ore passate al simulatore non erano sufficienti a contrastare la situazione contingente: in sintesi è venuta meno, ancora una volta, quella sorta di "intelligenza collettiva" che guida un team. Ognuno ha fatto e agito singolarmente, senza informare il collega e agendo spontaneamente. Non era un team affiatato, ma un insieme di singoli professionisti, ignari l'uno di che cosa facesse l'altro. Questo mix, insieme all'errore umano del secondo copilota, ha determinato l'esito finale con le sue drammatiche conseguenze. Gli aerei moderni sono un condensato di tecnologia e di meccanismi sofisticati con un numero sempre maggiore di funzioni di controllo automatizzato, che aumentano la sicurezza, ma riducono il numero di informazioni fornite ai piloti, dando una visione incompleta di quanto sta accadendo. Sono mancate leadership, situation awareness, comunicazione, teambuilding: le famose non technical skills che determinano il successo di un team. Un insieme di abilità implementato negli anni proprio dai sistemi complessi, come l'aviazione, che, associate alle abilità tecniche, rendono più performanti gli ambienti lavorativi.

\section{"Il miracolo dell'Hudson"}

Passiamo adesso ad un altro caso d'incidente aereo, questa volta senza vittime o feriti, alla cui conclusione favorevo- 
le hanno contribuito sicuramente, oltre alle checklist, situation awareness, leadership e teambuilding. Tutti ricordiamo l'ammaraggio di fortuna di un Airbus A320-214 (volo Airways 1549) il 15 gennaio 2009 sul fiume Hudson, nella baia di New York [10]. L'aereo, decollato dall'aeroporto La Guardia di New York e diretto a Charlotte, ebbe una collisione aerea con uno stormo di volatili (bird strike) e, dopo neppure 10 minuti di volo, fu costretto a un ammaraggio di emergenza. Tutti i 150 passeggeri e 5 membri dell'equipaggio a bordo trovarono rifugio sulle ali del velivolo utilizzando le uscite di sicurezza e vennero tratti in salvo dalle numerose imbarcazioni accorse sul posto in quello che fu ribattezzato dalla stampa "il miracolo dell'Hudson" $[11,12]$. L'Airbus A320 incrociò uno stormo di oche del Canada, una specie di volatili di circa 5 chili. Due oche, finite ciascuna in un motore, bloccarono le turbine e spensero entrambi i motori. In genere le collisioni tra velivoli bimotore e stormi non hanno conseguenze perché il calore dei motori liquefa i volatili che entrano nel loro raggio d'azione. In caso di risucchio di materiale durante il volo, i motori, per evitare di esplodere o di scagliare frammenti nella carlinga, si spengono automaticamente e così avvenne anche nel caso in questione. In quella che può essere considerata sicuramente la peggiore delle situazioni in aviazione, lo spegnimento di tutti i motori di un velivolo, entrarono in azione quei meccanismi che non si attivarono nelle occasioni precedentemente considerate: comunicazione, situation awareness, leadership, teambuilding e impiego corretto delle checklist.

I due piloti coinvolti, Jeffrey Skiles e Chasley B. Sullenberger III, due veterani con migliaia di ore di volo alle spalle, non si erano mai incontrati prima, ma quella mattina, forti della loro esperienza, non trascurarono di rispettare tutte le procedure previste per l'equipaggio (la presentazione, il briefing sulle condizioni di volo e su eventuali criticità legate al viaggio) e compilarono insieme la checklist. In questo modo non solo furono sicuri del buon funzionamento delle apparecchiature strumentali del velivolo, ma riuscirono anche a fare squadra, trasformandosi da singoli professionisti competenti in un team affiatato. Infatti, appena si resero conto dell'avaria di entrambi i motori, decisero insieme le uniche cose corrette da fare: fare assumere i comandi al pilota più esperto e con angolo di visuale migliore per un atterraggio di emergenza non strumentale (Sullenberger) e permettere al secondo pilota di concentrarsi sulla configurazione da fare assumere all'aereo per l'eventuale crash e di ripassare le checklist utili per riaccendere i motori (Skiles era appena tornato da un corso sulle emergenze in volo e sapeva esattamente quali documenti consultare). La registrazione delle conversazioni in cabina durante quei pochi minuti (l'impatto si è verificato alle 15 e 27 e 36 secondi e l'ammaraggio è avvenuto alle 15 e 31) è impressionante per la calma e la naturalezza dei comportamenti che hanno mantenuto i due piloti. Anche la decisione di ammarare sull'Hudson fu la più naturale: con i due motori spenti non si poteva né ritornare a La Guardia, né proseguire fino al vicino aeroporto di Teterboro. All'eccezionalità della manovra corrisponde la semplice applicazione di procedure condivise e previste per casi del genere. In una situazione di emergenza, Sullenberger e Skiles erano diventati un team con un obiettivo comune: compiere un atterraggio di emergenza e mettere in salvo i passeggeri, utilizzando quanto era mancato nei casi considerati precedentemente: comunicazione, leadership condivisa, situation awareness e ricorso "automatico" a checklist appropriate. Le checklist si sono dimostrate uno strumento molto efficace nelle situazioni complesse, perché guidano a eliminare manovre inutili e ripetitive e, se utilizzate correttamente da tutti, concentrano l'attenzione sulle manovre essenziali. Compilare distrattamente una checklist equivale a non usarla, aumentando notevolmente il rischio di errori in Aeronautica, in Marina, ma anche in Medicina. La cabina di pilotaggio di un aereo è simile, per complessità di lavoro e molteplicità delle figure professionali coinvolte, a una Sala Operatoria. Anche in Sala Operatoria ci sono diverse figure con competenze specifiche e diversi gradi di responsabilità e la gestione delle risorse umane che operano nell'ambiente operatorio deve tenere conto di queste differenze, che potrebbero aumentare e collidere in caso di incidente o di evento stressante. La checklist di Sala Operatoria, opportunamente mutuata e modificata dall'esperienza aeronautica, ha una doppia utilità: la prima è quella di aumentare la sicurezza del paziente candidato a intervento chirurgico e la seconda è quella di "fare gruppo" mentre la si compila. Se ogni singolo componente del team partecipa attivamente alla compilazione della checklist, è favorita la formazione di un'unica squadra composta da diverse figure professionali, in cui la comunicazione è il fulcro che aumenta la sicurezza degli operatori coinvolti. Laboratorio di analisi e Sala Operatoria sono ambienti più simili tra loro di quanto si possa pensare, perché hanno in comune diversi aspetti:

- molti operatori devono interagire e devono svolgere molti compiti eterogenei

- le numerose interfacce rendono spesso la trasmissione dell'informazione incompleta, inaccurata o ritardata

- più operatori concorrono al completamento di un compito aumentando la frequenza con cui tali compiti sono interrotti.

\section{Il $\mathrm{C}_{4} \mathrm{I}$ serve anche in sanità?}

D'altra parte, da epoca immemorabile i militari hanno vinto battaglie e guerre se avevano buone comunicazioni. Per loro l'accuratezza della trasmissione dell'informazione è sempre stata fondamentale; un'interruzione o un ritardo, anche se brevi, possono causare effetti catastrofici come la sconfitta $o$ la perdita di uno o più soldati. Proprio in ambito militare 
è stato coniato molti anni fa l'acronimo $\mathrm{C}_{4} \mathrm{I}$, che condensa in tre caratteri cinque strumenti che possono consentire al comandante di una divisione di affrontare "la nebbia della battaglia" e al direttore di un'Unità Operativa di affrontare "la nebbia della diagnosi". I cinque strumenti sono: Command (Direzione), Control, Communication, Computer, Intelligence; il web documenta quanto sia utilizzato il $\mathrm{C}_{4} \mathrm{I}$ (nel dicembre 2011 gli hits per $\mathrm{C}_{4} \mathrm{I}$ su Google erano $1.340 .000 \mathrm{e}$ quelli relativi al settore Sanità 1.180.000; nel maggio 2013 sono diventati, rispettivamente, 1.350 .000 e 1.890 .000 ).

- Command and control $\left(\mathrm{C}_{2}\right)$. Il direttore esercita le funzioni di direzione e controllo per raggiungere gli obiettivi assegnati attraverso un insieme di personale, strumentazione, sistemi e collegamenti informatici.

- Computing and communication. Costituiscono il supporto indispensabile del $\mathrm{C}_{2}$ e dell'intelligence. L'intelligence militare è il prodotto delle azioni di raccolta, integrazione, analisi, valutazione e interpretazione delle informazioni disponibili su Paesi stranieri.

Il modello $\mathrm{C}_{4} \mathrm{I}$ può fornire al direttore, come al comandante militare, la "situation awareness", la consapevolezza della situazione reale dell'organizzazione nell' ambiente che la circonda.

Comunicazione e informazione sono intimamente interconnesse e, anche se i nuovi dispositivi di comunicazione hanno reso più sfumata la distinzione tra le due attività, la comunicazione in Sanità ha cominciato a essere studiata solo di recente. Le inefficienze in questo ambito sono grandi e note, ma poco è stato scritto e fatto per porvi rimedio se non da un numero ristretto di ricercatori come Enrico Coiera, che ha studiato l'argomento prima nel Regno Unito e successivamente in Australia [13-15].

Il repository più grande di informazioni cliniche che risiedeva fino a poco tempo nella testa degli operatori comincia a spostarsi nella fitta rete di conversazioni che collegano gli individui e la comunicazione è sempre più importante perché l'esperienza biomedica non risiede più nei singoli, ma nel sistema. L'importanza di sapere è progressivamente sostituita da sapere dove trovare e il clinico deve essere sempre di più "connesso" ad altri clinici e a "macchine", in modo da condividere conoscenze e decisioni e formare team che affrontino "just in time" situazioni e pazienti.

Chi opera in sanità, a differenza di molti altri:

- si muove molto durante il lavoro (i medici, in particolare, hanno frequentemente occasione/necessità di spostarsi da un punto all'altro dell'ospedale);

- è spesso interrotto da colleghi, da telefonate o da cercapersone. L'operatore sanitario è quindi frequentemente costretto a gestire l'interruzione per poi tornare all'impegno precedente $\mathrm{o}$ a un'ulteriore interruzione più urgente (deve riprogrammare costantemente i numerosi impegni della giornata). Spesso chi interrompe il lavoro del collega non realizza quali inconvenienti questo può produrre.
In Sanità spesso l'attività non è singola ma in team, che opera nello stesso gruppo o in gruppi complementari (ad esempio, internisti, specialisti, radiologi, laboratoristi, chirurghi, anestesisti); questo richiede la comunicazione all'interno dello stesso team o tra operatori di team diversi.

Il diffondersi dei mezzi di comunicazione asincroni come i fax e le e-mail, che consentono di non prendere visione immediatamente della comunicazione e di farlo in un momento successivo più favorevole, ha ridotto il ricorso alla comunicazione immediata, che permette un'interazione più efficace. Dal punto di vista pratico è difficile fissare un appuntamento quando le agende sono così fitte e non sempre pianificabili esattamente e quindi spesso non si vuole perdere l'occasione quando si presenta.

\section{Che cosa succede quando una procedura è interrotta?}

Anche negli spazi più controllati e "concentrati" come la Sala Operatoria, le interruzioni sono frequenti e possono causare effetti negativi ed errori. Non sempre l'interruzione aumenta la durata di una procedura sanitaria; in qualche caso, addirittura, la diminuisce [16]. Questo può essere dovuto al fatto che l'operatore "accelera" per compensare per il ritardo eventuale semplificando le azioni che intraprende. Quindi, non tutte le interruzioni sono pericolose e, in qualche caso, sono tollerate; possono consentire di gestire delle urgenze, di completare altre procedure quando l'interruzione avviene da parte di un collega necessario per completare altre attività. Autori come Berg et al hanno recentemente segnalato che tali interruzioni sono percepite come dannose dai clinici, anche perché sono state associate a un aumento del carico di lavoro [17, 18].

Il Laboratorio clinico rappresenta un caso del tutto particolare in questo contesto e per il quale non esiste praticamente letteratura. D'altra parte, il Laboratorio processa, per sua natura, un elevato numero di messaggi: tipicamente riceve i messaggi con la richiesta di esami e genera dei referti, quindi altri messaggi, per il medico richiedente (e per il paziente).

Il Laboratorio tradizionale aveva uno staff piccolo con pochi problemi di comunicazione interpersonale, ma anche verso l'esterno (ogni ospedale disponeva di un proprio Laboratorio). Il processo del consolidamento ha aumentato il numero di operatori che compone uno staff e ha allungato la distanza tra il Laboratorio e i clinici che possono essere anche in un ospedale diverso o comunque distanti.

Questa nuova organizzazione ha favorito e favorisce la trasmissione asincrona della comunicazione.

\section{Gli smartphone}

Oggi possiamo comunque dire che l'introduzione dirompente degli smartphone (SM) ha modificato drasticamente la 
comunicazione in qualunque genere di business e non ultimi Sala Operatoria e Laboratorio. In pratica gli smartphone nascono come "fusione" di Personal Digital Assistant, le agende elettroniche, che hanno un avuto un certo successo anche in Sanità, e telefoni mobili, che hanno avuto uno straordinario successo universale. La possibilità degli SM di accedere a internet, archiviare file e scaricare programmi (le applicazioni, app) ha portato a un successo di dimensioni ancora più clamorose [19]. Lo smartphone consente di passare dalla $E$-medicine alla $U$-medicine, un qualcosa di molto diverso dalla Medicina elettronica. Possedere le $4 \mathrm{U}$ significa, infatti, essere ubiquitario (accesso all'informazione non limitato dal tempo e dallo spazio), unico (conoscenza precisa delle caratteristiche e della sede dell'individuo che consente di personalizzare le informazioni, all'unisono (l'individuo vuole un singolo riferimento, non una dozzina di banche dati, spesso in conflitto tra loro) e universale (l'utente vuole un unico dispositivo che serve da telefono, browser, PDA, macchina fotografica, riproduttore di musica, GPS) [20].

Anche se gli smartphone sono impiegati in Laboratorio e potenzialmente possono rivoluzionarlo [21], non sono ancora impiegati al loro pieno potenziale [22].

Cominciano a esserci segnalazioni nell' ambito delle attività commerciali e industriali sui "pericoli” degli smartpho$n e$. Non c'è dubbio che gli SM connettono (connect) il professionista (ad esempio, banchiere, avvocato, commerciante, medico) all'organizzazione ma non siamo sicuri che li interessino (engage) alla mission della stessa. È esperienza comune di avere osservato partecipanti a qualche riunione che sono fisicamente presenti ma mentalmente assenti e concentrati in altre attività, sempre di più impegnati in quella che gli anglosassoni chiamano la "smartphone prayer" sotto il tavolo [23]. Il problema opposto è quello del sovra engagement spesso alimentato dalla prodigiosa capacità degli SM di mantenere connessi per 24 ore al giorno 365 giorni l'anno. Questo approccio, oltre a essere causa frequente di burn out, è dannoso per l'organizzazione, perché può portare a pensare che tutti i collaboratori debbano seguire lo stesso ritmo di lavoro. Spesso i "super-connessi” sono poco efficienti anche se sono sempre occupati e ricorrono al controllo diretto piuttosto che a obiettivi condivisi. Un aspetto interessante dell'iperconnettività è che ha dei gravi effetti collaterali: riduce l'attenzione, la concentrazione e la profondità nella valutazione dei problemi. È stato stimato che occorrono in media 15 minuti per riorientare l'attenzione dopo avere ricevuto una e-mail e che la produttività cala dal $20 \%$ al $40 \%$ quando i compiti sono svolti in parallelo e non in serie. In modo molto provocatorio Judith Mc Cormick et al concludono [23] 'You've got people sending copious amounts of e-mails when they could pick up the phone or turn around and talk to the person sitting behind them". E ancora "Too much information kills communication".
L'organizzazione può averne un danno perché gli SM, che avevano lo scopo di condividere le informazioni, collaborare e coordinare, possono eliminare la delega. Prima, almeno durante le vacanze, il direttore delegava ai suoi collaboratori, ma con l'avvento degli SM continua a prendere le decisioni.

Il modello più corretto potrebbe essere quello del Connesso Dinamico, che nel corso della giornata ha delle parti ad alta connettività e delle parti a bassa connettività secondo le necessità e ha la capacità di sconnettersi e di ricaricare le batterie fisiche, mentali ed emozionali.

\section{Conclusioni}

In conclusione:

- può essere irrilevante per l'organizzazione premiare il tempo in cui un dipendente resta in contatto se non è realmente "engaged" nell' attività dell'organizzazione;

- se il contatto costante porta a interruzioni costanti, il nostro cervello si distrae e affronta problemi complessi in modo poco efficace;

- l'iperconnettività può indurre una sorta di dipendenza al "Crackberry", che può risultare, alla fine, demotivante.

Siamo convinti che informazione e comunicazione sono egualmente indispensabili in reparto, in Sala Operatoria e in Laboratorio, ma non sono sufficienti. Molto più efficace è il modello $\mathrm{C}_{4} \mathrm{I}$, che può fornire al dirigente, come al comandante militare, la "situation awareness", la consapevolezza della situazione reale dell'organizzazione nell' ambiente che la circonda. Il dirigente dovrà integrare la "situation awareness" con quello che sa sul personale (integrità, competenza, esperienza, carattere, integrazione con i colleghi, capacità di gestire lo stress) e con quanto sa su elementi "incerti" come adeguatezza e affidabilità delle forniture, trend delle richieste di esami ricevute dal Laboratorio, necessità di introdurre nuovi esami e di dismetterne di vecchi.

Ricordiamoci di quello che è successo a Tenerife, Rio de Janeiro, Hawaii e non illudiamoci che a noi non possano capitare incidenti perché siamo "bravi". Alcuni dei piloti più esperti della loro generazione hanno gettato reputazione, carriera e vita in pochi minuti perché non hanno comunicato in modo efficace.

\section{Conflitto di interesse Nessuno.}

\section{Bibliografia}

1. Weik KE (1990) The vulnerable system: an analysis of the Tenerife air disaster. J Manag 3:571-593 
2. https://en.wikipedia.org/wiki/Tenerife_airport_disaster [Accesso 10 luglio 2013]

3. Gawande A (2009) The checklist manifesto. Metropolitan Books, New York

4. Meilinger PS (2004) When the fortress went down. Air Force Mag 10:78-82

5. Haynes AB, Weiser TG, Berry WR et al (2009) A surgical safety checklist to reduce morbidity and mortality in a global population. N Engl J Med 360:491-499

6. de Vries EN, Prins HA, Crolla R et al (2010) Effect of a comprehensive surgical safety system on patient outcomes. N Engl J Med 363:1928-1937

7. Dorizzi R (2003) Gli errori in medicina: cosa ci insegna il disastro del sommergibile USS Greenville. Verona Medica 38:32-33

8. Roberts HK, Tadmor CT (2002) Lessons learned from nonmedical industries: the tragedy of USS Greenville. Qual Saf Health Care 11:355-357

9. Wise J (2012) Air France 447 and the limits of aviation safety. Popular Mechanics, 9 July. http://www.popularmechanics. com/technology/aviation/crashes/air-france-447-andthe-limits-ofaviation-safety-104875 [Accesso 10 luglio 2013]

10. Langewiesche W (2010) The miracle plane crash-landing on the Hudson river. The Sunday Times, 10 febbraio. http://www. timesonline.co.uk/tol/news/world/us_and_americas/ article7013967.ece [Accesso 10 luglio 2013]

11. Eisen LA, Savel RH (2009) What went right. Lesson for the intensivists from the crew of US airwais flight 1549. Chest 136:910 917
12. http://www.nytimes.com/interactive/2009/01/15/nyregion/ 20090115-plane-crash-970.html?_r=0 [Accesso 10 luglio 2013]

13. Coiera E (2006) Communication systems in healthcare. Clin Biochem Rev 27:89-98

14. Coiera E (2004) Four rules for the reinvention of health care. BMJ 328:1197-1199

15. Coiera E (2012) The science of interruption. BMJ Qual Saf May 21:357-360

16. Westbrook JI, Coiera E, Dunsmuir WTM et al (2010) The impact of interruptions on clinical task completion. Qual Saf Health 19:284-289

17. Berg LM, Källberg AS, Göransson KE et al (2013) Interruptions in emergency department work: an observational and interview study. BMJ Qual Saf 22:656-663

18. Coiera EW, Jayasuriya RA, Hardy J et al (2002) Communication loads on clinical staff in the emergency department. MJA 176:415-418

19. Phillippi JC, Buxton M (2010) Web 2.0: easy tools for busy clinicians. J Midwifery Women's Health 55:472-476

20. Pitt LF, Parent M, Junglas I et al (2011) Integrating the smartphone into a sound environmental information systems strategy: principles, practices and a research agenda. J Strateg Inf Syst 20:27-37

21. Lippi G, Plebani M (2011) Laboratory applications for smartphones: risk or opportunity? Clin Biochem 44:273-274

22. Abeynaike L (2012) Qualitative study on the applications of smartphones in medical research. J MTM 1:19-23

23. Mc Cormick JS, Dery K, Kolb DG (2012) Engaged or just connected? Smartphones and employee engagement. Organ Dyn 41:194-201 